\title{
Cybernetics of the Mind: Learning Individual's Perceptions Autonomously
}

Plamen Angelov, Xiaowei Gu, Jose Iglesias, Agapito Ledezma, Araceli Sanchis, Oscar Sipele, Ramin Ramezani Plamen Angelov and Xiaowei Gu are with the School of Computing and Communications, Lancaster University, LA1 4WA, UK; e-mail: p.angelov@lancaster.ac.uk

Jose A. Iglesias, Agapito Ledezma, Araceli S. de Miguel and Oscar Sipele are with the Informatics Engineering Department, Carlos III University, Madrid, Spain, e-mails: < jiglesia, ledezma, masm, bsipele >@inf.uc3m.es Ramin Ramezani is with Computer Science department, UCLA; e-mail: raminr@ucla.edu

\begin{abstract}
In this paper, we describe an approach to computational modelling and autonomous learning of the perception of sensory inputs by individuals. A hierarchical process of summarization of raw data with heterogeneous nature is proposed. At the lower layer of the hierarchy, the raw data autonomously forms semantically meaningful concepts. Instead of clustering based on the visual or audio similarity, the concepts are being formed at the second layer of the hierarchy based on the observed physiological variables (PVs) like heartbeat, skin conductance, etc. and mapped to the emotional state of the individual. Wearable sensors were used in the experiments. Methodologically, we used the recently introduced Empirical Data Analytics (EDA) computational framework and the data partitioning method within EDA to form the data clouds (cluster-like formations with no pre-defined shape) autonomously and used AnYa type of IF-THEN fuzzy rule-based models to describe the mapping of the observable PVs to the emotional states. Multi-modal typicality distributions, which have properties like pdf and indicate the empirical likelihood distributions of the data, can be further derived without making prior restrictive assumptions traditionally required in statistical approaches. The quality of the classifier is evaluated by a confusion matrix and classification rate/precision. The experimental results are very encouraging: for this extremely complicated problem, we got $74.58 \%$ correct classification results without any pre-training.
\end{abstract}

Keywords- computational modelling; autonomous learning; human perception; Empirical Data Analytics (EDA); AnYa type fuzzy rule.

\section{Introduction}

The nature of perception of the sensory inputs has been a subject of many studies [1], [2]. One aspect of these works is the psychological one [3], [4]; another one is the subjective mapping to fuzzy sets [5]. Some studies attempted to introduce computational modelling aspects [6]. Human emotions are very hard to analyse due to the 
individuals' differences. In the recent decades, the machine learning techniques have been used in studying and recognizing human emotions [7]. Different studies have been conducted to detect individuals' feeling of stress using the computational classification/recognition models based on different features or to automatically recognize people's personalities and their interaction styles based on their Facebook profile pictures [8]. Studies on personality recognition based on people's essays has also been conducted [9].

In this paper, we introduce and describe an approach to computational modelling of the learning process including the role of the individual's perception. Our approach is different from the existing studies on cognitive neuroscience where the learning is considered at a neuron and brain elements (visual cortex, hypothalamus, etc.) level [10], [11]. It is also significantly different from the large body of literature on perception in a psychological context where the studies are qualitative and descriptive [1]-[4]. Instead, we adopt a systems-theory-based (cybernetic) approach to the problem focusing on the macro level information processing when the psychological, biochemical and bioelectrical aspects are deliberately ignored. Instead, the focus is on the individual's perceptions observed through easy to measure (including with wearable sensors) physiological variables (PVs) and the brain of the individual being considered as a system, the state of which is of significance, not the internal components' interactions. The proposed approach is generic in terms of all five types of sensory inputs (visual, auditory, tactile, aromatic and digestive). However, we limit our experiments to visual and auditory inputs only and we consider similarity based on the PVs and, respectively emotions, not in terms of visual or audio similarities. In our study, we consider the following easy to observe and measure PVs: heart beat and skin conductance. The proposed approach considers a layered hierarchical structure with the lower layer mapping and integrating the sensory inputs expressed (and measured) by observable PVs into generalized, aggregated data clouds. We consider this a sub-conscious process and, thus, unsupervised learning to take place. At a higher hierarchical level, these data clouds get semantic meaning and become concepts, such as "positive", "negative". In general, the granularity of the semantic categories can be higher, e.g. "beach", "mountain", "motorway", "train”, “dog”, “door”, "gun” etc. or more complex, composite concepts such as "office", "war scene", "market place", etc. In addition to this hierarchically layered scheme we consider the conscious decision-making block which may have a cognitive or deliberative feedback and is influenced by goals and results in (re-)actions of the individual. It further maps these semantically meaningful generalized concepts into emotions by a self-developing linguistically transparent AnYa type rule-based model [12]. In this paper we aim proof of concept and, therefore, limit our study to a steady state case and simple, not composite 
concepts (e.g. "positive" and "negative") using images and music only and not active decision making cognitive feedback. We are aware, however, that in reality, a dynamic model, e.g. of the type of hierarchical Kalman filters type is more suitable; this will be studied in further works. For example, in [10], emotions were not considered and the study was limited to a single sensory (video) input. According to the proposed CyberMind approach, autonomous learning process maps the observable PVs which represent individual- and context/temporal- specific emotions. This corresponds to the subconscious manner our emotions are being associated and aggregated into types of subconscious reactions. That means that an individual does not need to do anything to produce these outputs or, something more, usually can hardly hide them even if wanted to (they are predominantly objective). Similarly, to a lie detector, essentially, we offer a "emotions" mapper/reader/model. This specific role of the emotions is very important in regards to understanding the nature of unsupervised learning in individuals. Furthermore, we propose a transparent IF THEN type linguistic model of AnYa type. It ca be used to classify unseen images and thus predict the emotional reaction of an individual during a given session of the experiment. We, further, propose and perform an experiment with wearable non-intrusive devices such as heartbeat and skin conductance sensors for identifying the emotions. As a result, we were able to learn and predict in $74.58 \%$ of the cases the emotional perception of specific images by an individual within a given session. The proposed approach contributes to the better understanding of the nature of perception and learning. It also offers opportunities to build tools and devices that can be useful for advertisement, medicine, entertainment, predicting reactions of professionals to specific situations, e.g. firefighters, police personnel, etc.

The remainder of the paper is organized as follows. In section II we introduce the concept of the proposed approach. The methodology is described in section III. Section IV describes the experimental study as well as analysis of the results, finally, section $\mathrm{V}$ concludes the paper.

\section{The Concept of the Proposed Method}

In this section, the concept of the proposed approach will be described. We start with the observation that the nature of individual's perceptions and learning are neither clearly probabilistic nor deterministic. A simple example that illustrates this is if we consider a memorable scene we may have seen just once in our life or think of the efforts it will take to learn a foreign language (say, as an example, for the authors of this paper, Japanese). Learning is clearly linked with emotions, associations and is not simply a matter of "repeat $n$ times". Associations in learning have been studied earlier from psychological point of view, e.g. in [10]. In computational intelligence, the fuzzy set 
theory [5] was proposed to represent mathematically the subjective preferences and claims to deal with perception. However, traditional approaches require a priori defined or assumed type of the membership functions (in the case of fuzzy sets theory [5]) or probability distribution functions (pdf) [13] in the probabilistic approach. In addition, the probabilistic approach also requires "large enough" (or theoretically infinite) amount of data, their independence/orthogonality to build a credible model.

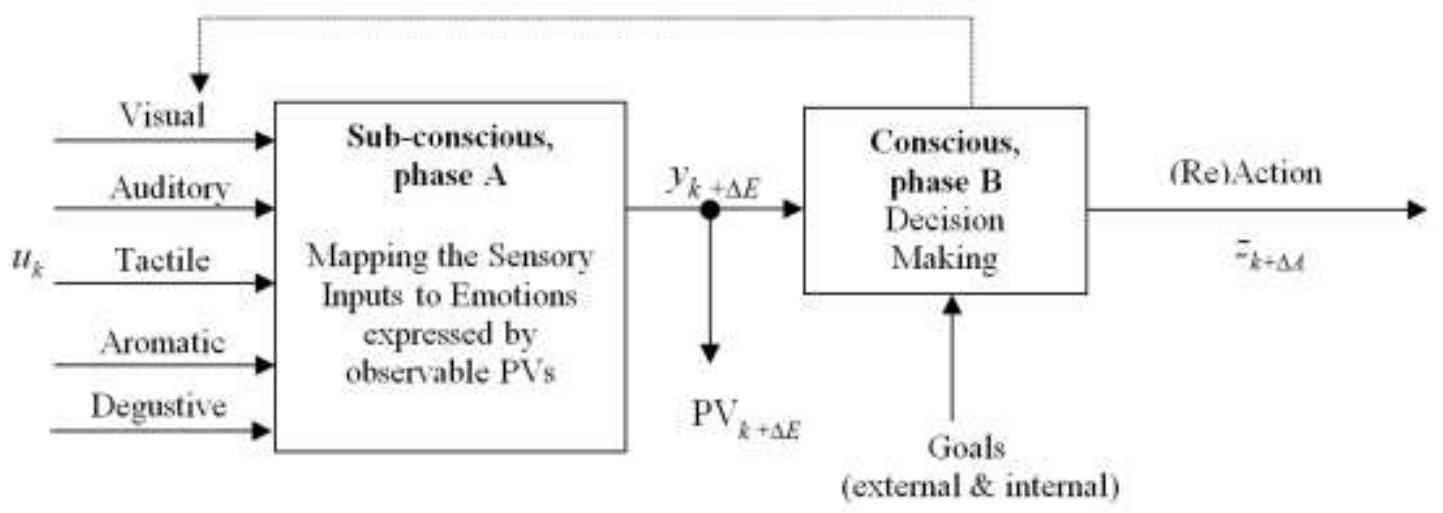

Fig. 1. A system-theory-based view of the mapping of sensory inputs to emotions (and further to actions)

In this paper, we propose to build a macro-level cybernetic model of the mapping of the sensory input to the emotions represented by the observable PVs. We consider this as a step (phase A, Fig. 1) towards a better understanding and as a (sub-conscious) part of the more complex problem of the process of individual's learning.

We will focus on phase A and will only consider (without limiting the generality of the concept) visual and audio inputs $\left(u_{k}\right)$ and heart beat rate (bmp) and skin conductance (in micro Siemens, $\mu \mathrm{S}$ ) as observable PVs (in this study instead of real visual similarity between images or audio similarity between music, it is a $2 \mathrm{D}$ vector, $y_{k+\Delta E}=$ $\left[\begin{array}{c}b m p \\ \mu \mathrm{S}\end{array}\right] k+\Delta E$ ); where $\Delta E$ denotes the delay in expressing emotions to a sensory (in this study, visual) input (usually this is reported to be less than $10 s$; obviously, this is person-specific and different for different types of sensory inputs); $\Delta A$ denotes the delay in (re-)actions which may be longer or may never take place and is not a subject of this paper. The cognitive feedback (dotted line in Figure 1) exists in real life in most of the cases when we are awake and conscious, but we will ignore it and we will also consider steady state only (in reality there are also transients related to the fact that we continuously get different sensory inputs).

We argue that everything else is being learned through experience and we propose a method to mathematically model this process within the systems theory and cybernetics framework. To the best of our knowledge, this is the first such comprehensive model within the cybernetics framework. We also argue that the learning of people is 
always linked with emotions and a cognitive feedback in addition to the emotions and, thus, disposition (positive or negative) to learning. However, it is very often (especially in adults) of unsupervised type of learning in the broader sense that no "external teacher" or external feedback of stimulus may be present and still we can continue to learn autonomously and dynamically evolve our own understanding of the world [14]. This hypothesis has strong links with the ART concept by Carpenter and Grossberg in regards to the threshold and arousal when new information is presented [11].

In this paper, we consider a layered hierarchical structure of learning and "making sense" from the sensory inputs as depicted in Fig. 2.

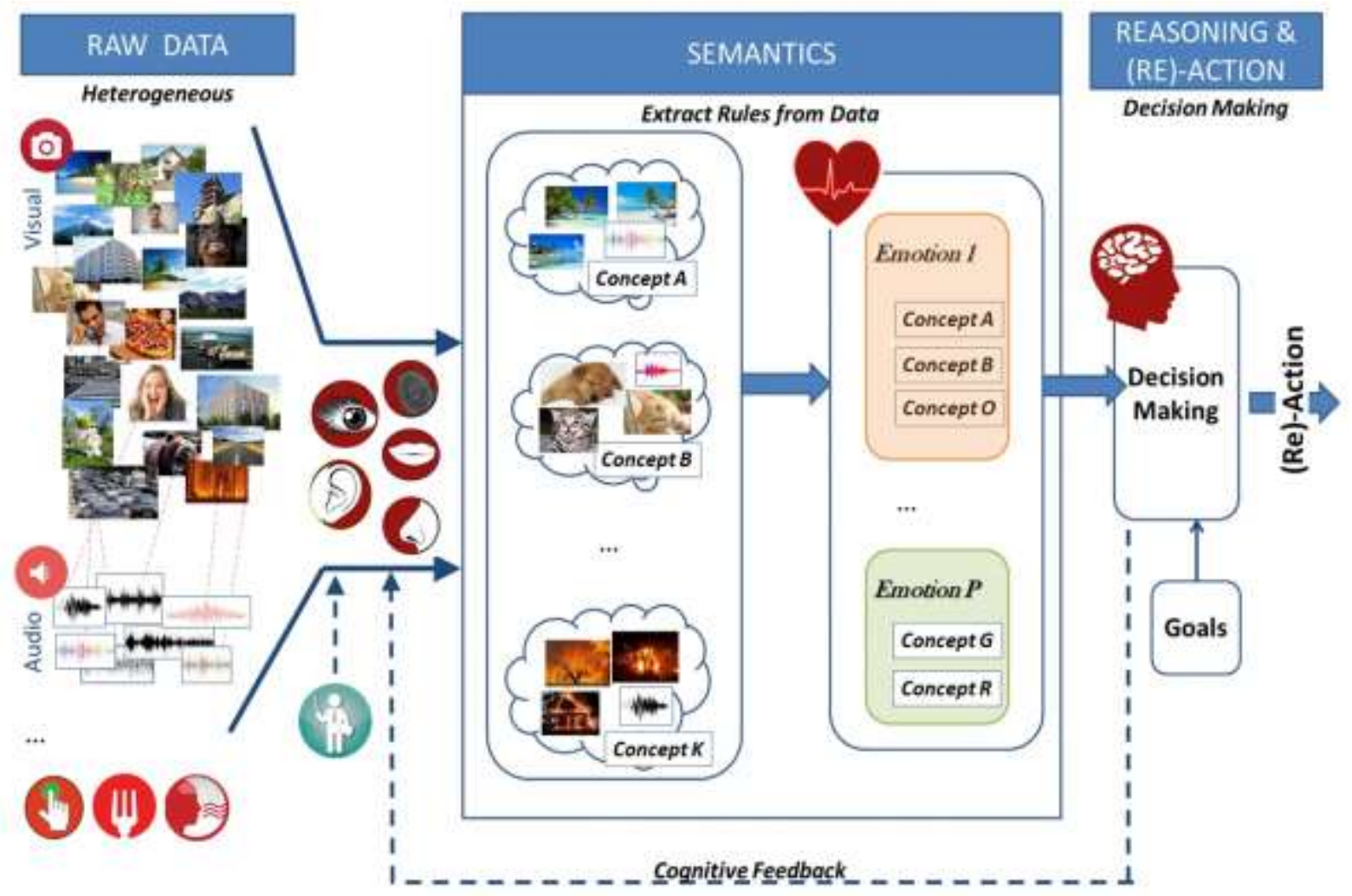

Fig. 2. A layered hierarchy of making sense from data (CyberMind architecture)

In particular, we consider, a three layered structure $(\mathrm{L}=3)$, where Layer 1 corresponds to forming relatively small number of concepts with clear semantic meaning, aggregating and integrating the raw multi-modal data streams (visual, auditory, aromatic, etc.). Examples of such concepts can be "good", "bad", "door", "window", "flowers", "beach", "motorway", etc. It is also possible that more complex, composite concepts are also formed such as "market place", "war", "hospital", "office", etc. The process of forming concepts based on clouds of data with similar properties is not considered in detail in this paper, but this can be automated as described in [15]. This 
process can be done with a cognitive feedback (the dotted line in Figure 1) or in some cases sub-consciously in a fully unsupervised manner when we associate some scene or music etc. with previously seen examples and concepts. Data clouds are like clusters, but are shape-free and form a Voronoi tessellation in the feature space [12], [14], [16]. This process of reducing the vast amount of raw data into a much smaller amount of concepts identifying meanwhile possible outliers [16], [17], Figure 1. In real life, examples of data clouds can be images of, sound from cars; dogs, etc. that we encounter throughout our lives.

According to the CyberMind architecture, at the second layer of the hierarchy these data clouds that represent semantic concepts can be linked into rules, story lines, graphs and labels can be assigned to them optionally. Our hypothesis is that by memorizing only the concepts (focal points of data clouds) plus outliers identified such as landmarks instead of the huge amount of raw data people they encounter in their live they are able to cope with this real life Big Data problem. In this way individuals transform this overwhelming amount of heterogeneous data into a manageable and individually specific subset of:

a) data clouds forming rules, labelled, organised into other structures for successive use in the conscious phase B for inference, prediction, decision making and (re)actions;

b) certain number of outliers that (for good or bad) has been memorised, too (mainly subconsciously).

Indeed, there are many studies which report that the people during sleep (unconsciously) revisit the raw data, reorganise it or various outliers come to attention [18], [19]. We are aware that better results may be achieved using EEG or ECG which are more informative but we deliberately do not consider these because we aim non- or minimum intrusiveness. Indeed, [20] demonstrated that different emotional states such as happiness, sadness and grief, anger, neutral state, etc. can be recognised based on physiological cues measured through heartbeat rate, skin conductance, oxygen level in the blood, etc. An example of a real experiment conducted in Carlos III University, Spain in September 2015 are depicted in Fig. 3, where two individuals' heart beat rate and skin conductance level were measured on one day in response to the same sequence of images and music/audio. This demonstrates the variability and the importance of the individual's specifics and the limitations of the "one size fit all" type of averages usually used in traditional statistical approaches where a response of a group of people is being modelled.

In this paper, we do not detail the decision making and (re-)action phase which is conscious and follows the process at the second layer of the hierarchical layered structure we propose. Instead, we focus on the first phase, phase A, which may take place sub-consciously (and, thus, in an unsupervised manner) where perceptions of 
individuals are being formed and emotions are expressed. We further simplify the real life case by considering only simple (not composite) concepts (e.g. "positive" and "negative") and only steady state (not transient and dynamic) model. We offer a hint how this can be addressed in further research.

\section{Methodology}

In this section, we will introduce the theoretical basis employed by the proposed method for learning and modelling individual's perceptions.

\section{A. Empirical Data Analytics}
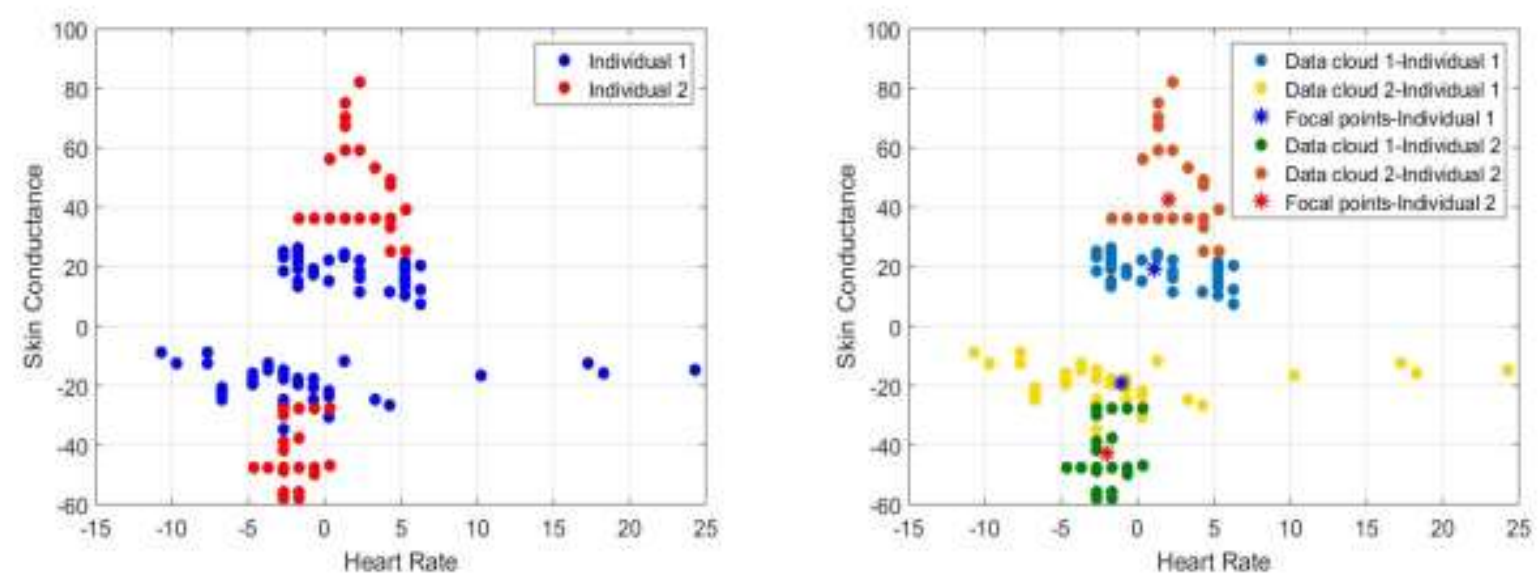

Fig.3. Observed PVs of the individual 1 and individual 2 Fig.4. Data clouds formed based on the PVs in Fig. 3

Due to space and other limitations, we will not introduce the details of the EDA (Empirical Data Analytics) computational framework. Its main distinctive feature is that without the need for prior assumptions about the data distributions, amount of data or their (in)dependence or even their randomness or deterministic nature we can directly (without iterative cycles and loops, in a recursive manner) derive multi-modal typicality distributions which have same properties as the pdf, e.g. integrate to 1 [21]-[23]. With the EDA framework, one can partition the collected experimental data and group into data clouds [16]. These can then be labelled, for example, "Emotional state 1", "Emotional state 2" and so on, see Fig. 4, where the values are centred ( subtracted mean). In the rest of this paper, ES 1 represents the emotional state 1 and ES 2 represents the emotional state 2.

Furthermore, the continuous global typicality can be build, see Figs. 5 and 6 , which is a measure of the likelihood and is fully based on the observed data samples without making any prior assumptions, i.e. the distribution of the data. In fact, if the distance metric that is used is Euclidean it takes Cauchy form which is quite similar to the Gaussian function [22], [24]. 


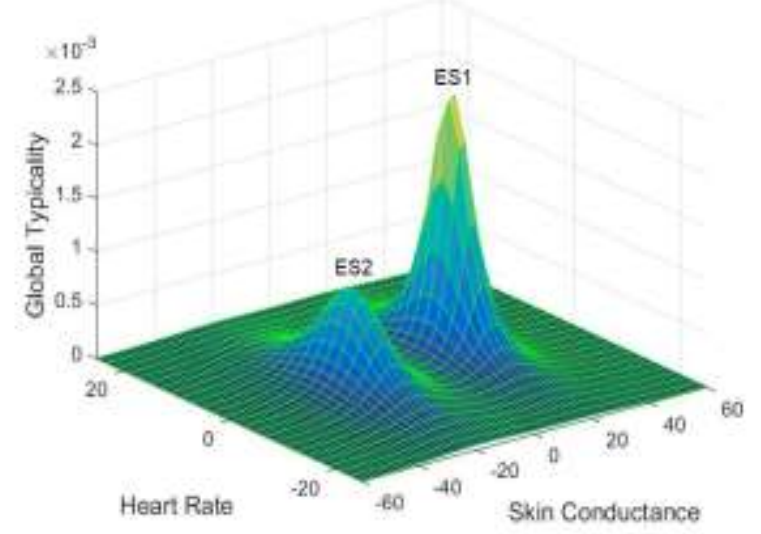

(a) Individual 1

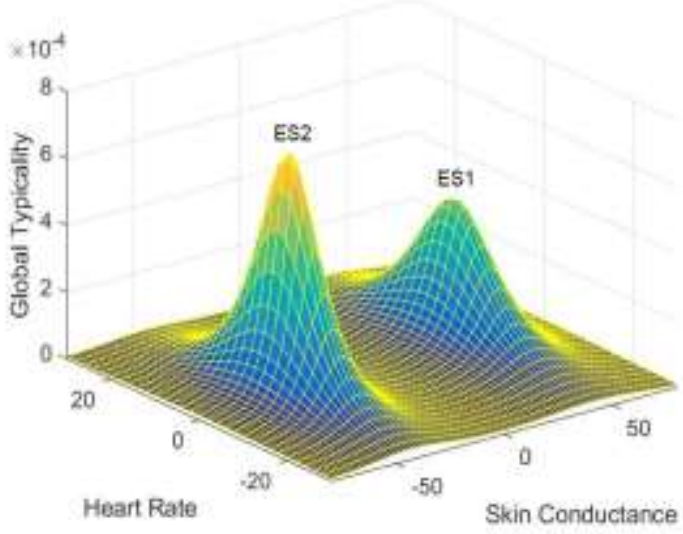

(b) Individual 2

Fig. 5 The $3 D$ continuous global typicality built based on individuals' data as described in [16],[23]
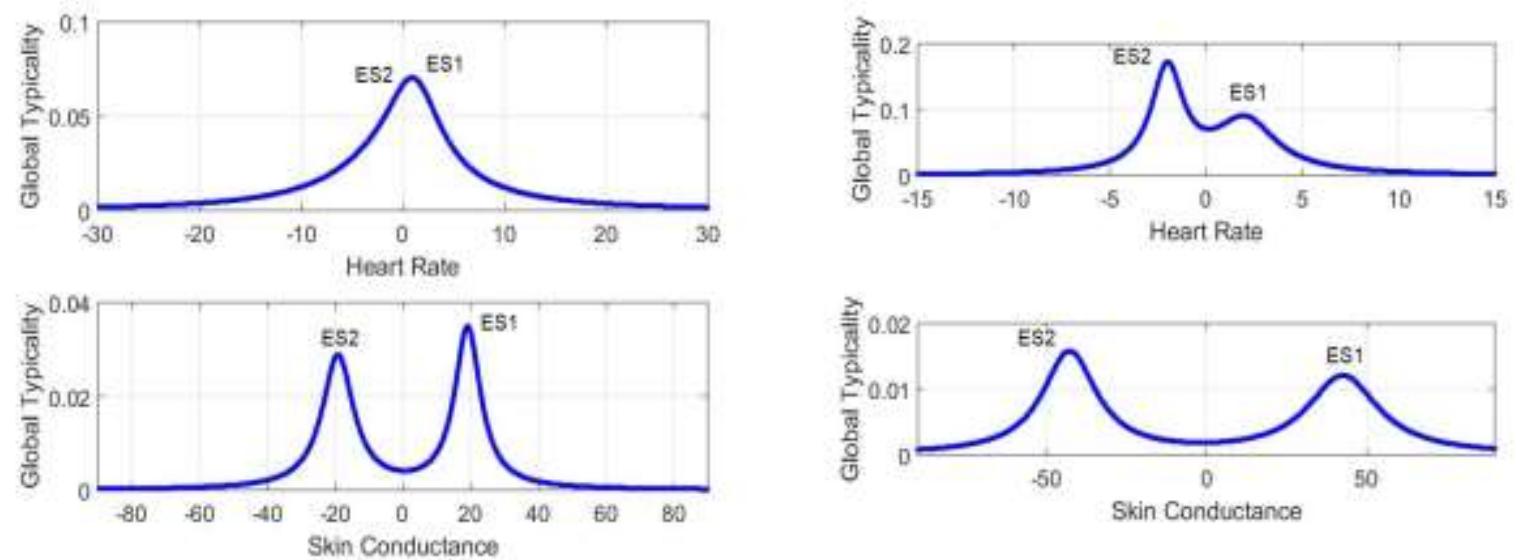

(a) Individual 1

(b) Individual 2

Fig. 6 The $2 D$ continuous global typicality built based on individuals' data

\section{B. Fuzzy rule-based classifier of 0 order AnYa type:}

AnYa is a new type of FRB systems introduced by Angelov and Yager [12]. Compared with the Mamdani and Takagi-Sugeno type FRB systems, the antecedent part of the AnYa type FRB system is revised and simplified. Using AnYa type fuzzy rule, we can model individual 1 and individual 2's reactions as:

$$
\text { Individual 1: }\left\{\begin{array}{l}
I F\left(\left[\begin{array}{c}
b m p \\
\mu \mathrm{S}
\end{array}\right] \sim\left[\begin{array}{c}
1.0625 \\
19.1375
\end{array}\right]\right) \operatorname{THEN}(E S \text { 1) } \\
I F\left(\left[\begin{array}{c}
b m p \\
\mu \mathrm{S}
\end{array}\right] \sim\left[\begin{array}{c}
-1.0625 \\
-19.1375
\end{array}\right]\right) \operatorname{THEN}(E S \text { 2) }
\end{array}\right.
$$




$$
\text { Individual 2: }\left\{\begin{array}{l}
I F\left(\left[\begin{array}{c}
b m p \\
\mu \mathrm{S}
\end{array}\right] \sim\left[\begin{array}{c}
2.0250 \\
42.6500
\end{array}\right]\right) \operatorname{THEN}(E S \text { 1) } \\
I F\left(\left[\begin{array}{c}
b m p \\
\mu \mathrm{S}
\end{array}\right] \sim\left[\begin{array}{c}
-2.0250 \\
-42.6500
\end{array}\right]\right) \operatorname{THEN}(E S \text { 2) }
\end{array}\right.
$$

where the data was centred using the subtracted mean.

With the fuzzy rules, one can model the individual's emotional reaction to similar images and music. This is, however, individual- and temporal/context- specific. That means, the models are not quite stable across various people and even for the same individual across different days - see Fig. 7, in which, are the first three days' experimental results of individual 3 and individual 4.
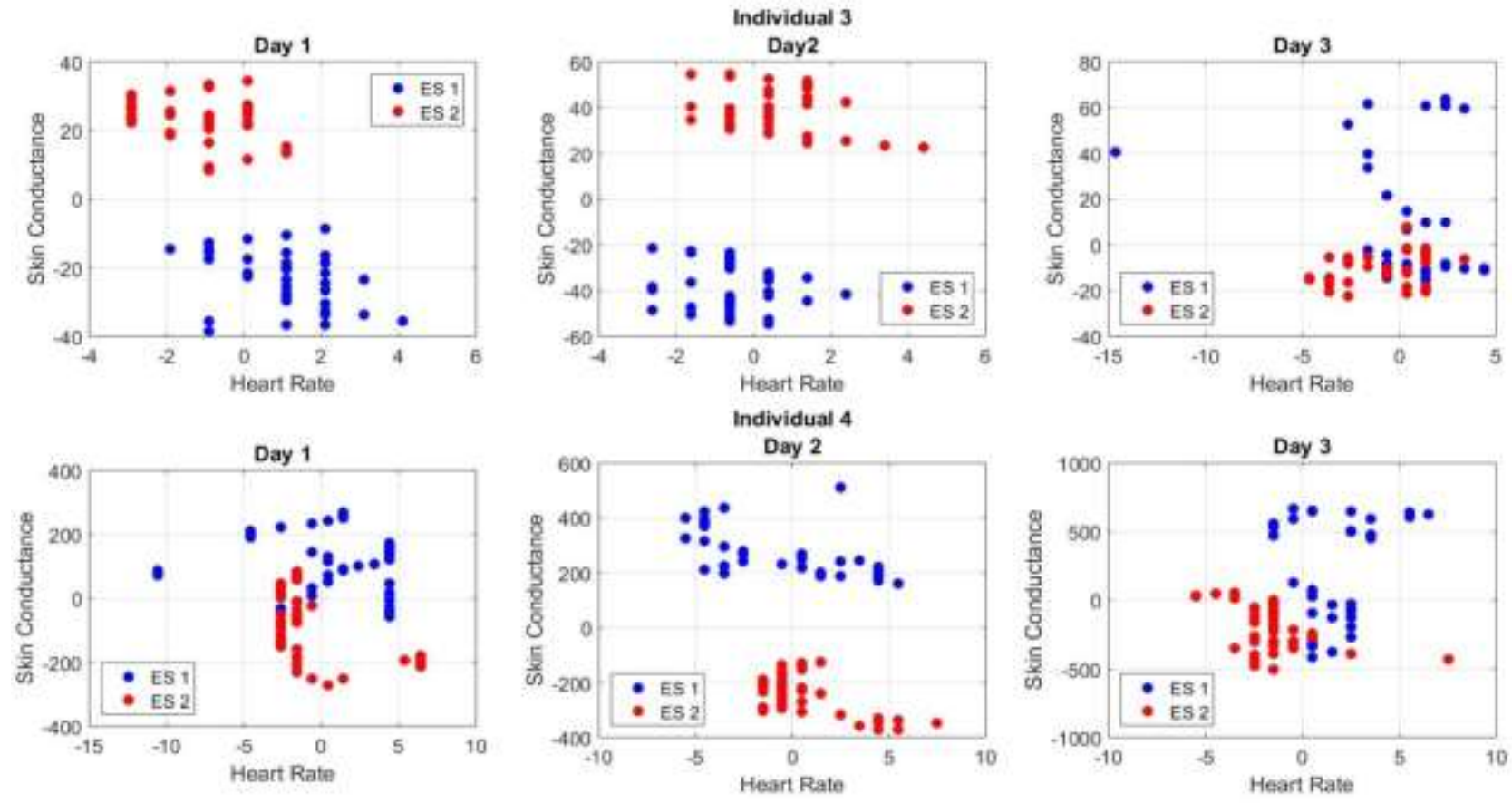

Fig.7. The experimental results of individual 3 and individual 4 for the first 3 sessions (days)

The reason is that people have different emotional history, background and inertia (a change of emotions does not immediately lead to a change in the observable PVs).

\section{Experimental Study and Analysis}

In this section, we will study the results of the real experiments conducted in Carlos III University in September 2015. In these experiments, 10 individuals were invited and the set of experiments lasted 5 days. On each day, researchers took the reading of the wearable sensors for measuring reaction (in terms of heart beat rate (in bpm) and skin resistance (in $\mu \mathrm{S}$ )) of the experimental subjects towards two sets of images and music (negative and 
positive). The images were different on each day. The two sets invoked two different types of emotions for each person and each session (day). Each session consisted of 4 sections and lasted for approximately 22 minutes, the main procedure of each experimental session as follows.

Section 1: 600 seconds; 1 pink image; no background music.

Section 2: 200 seconds; 40 positive images (5 seconds per image); background music: Kool and the gang - Celebration.

Section 3: 300 seconds; 1 pink image; no background music.

Section 4: 200 seconds; 40 negative images (5 seconds per image); background music: Slayer - Angel of Death.

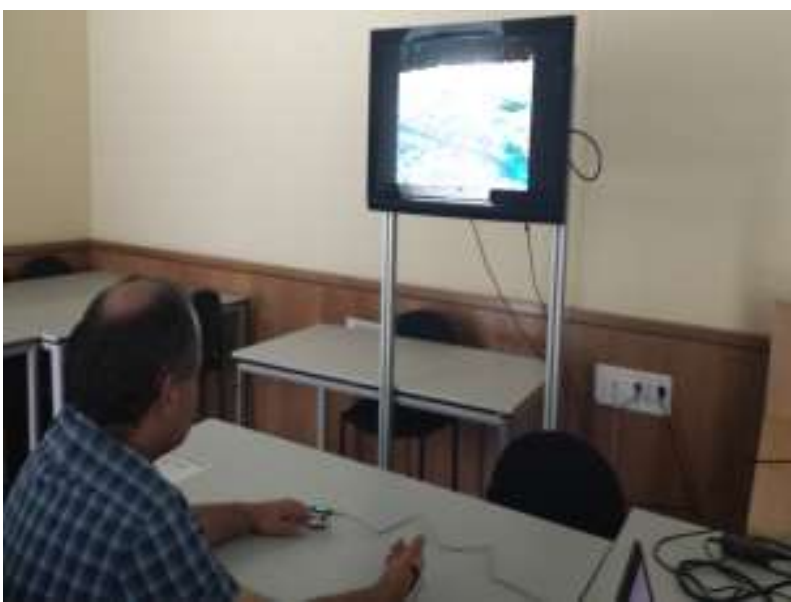

Fig. 8. A photo taken during one of the experiments

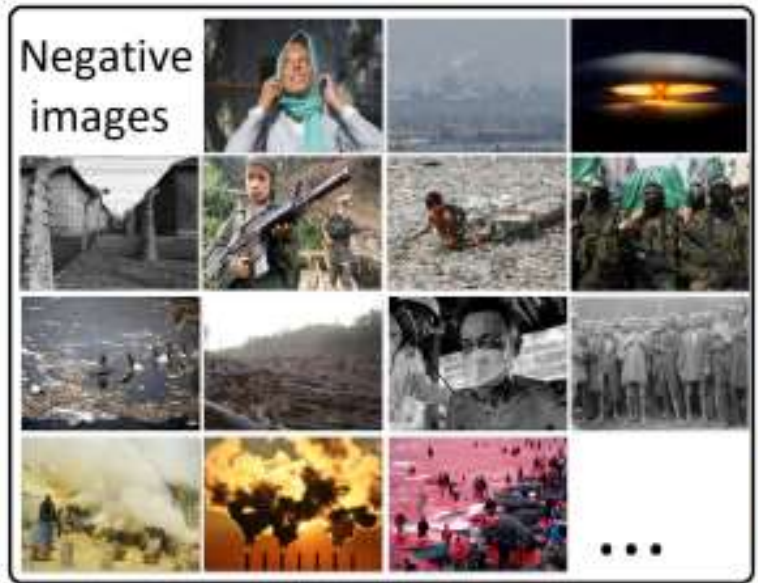

Fig. 10. Example images used in the experiments

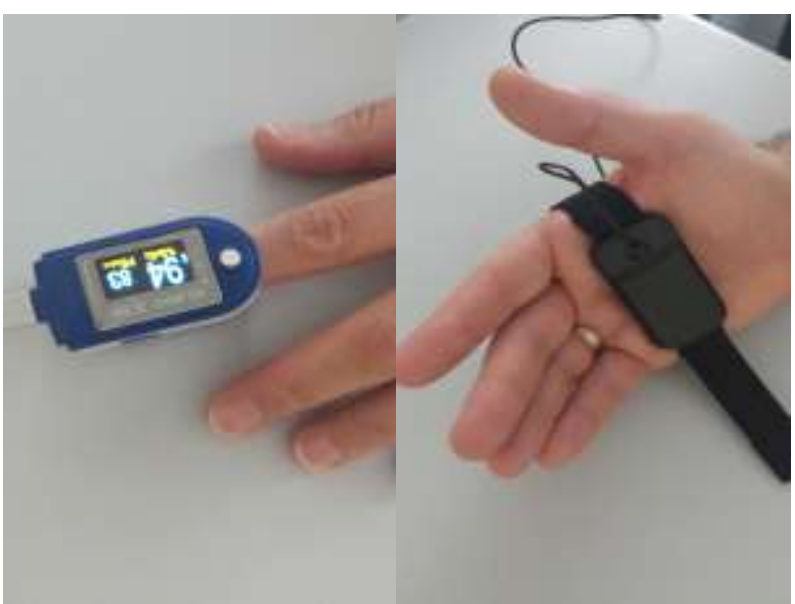

Fig. 9. Wearable sensors used in the experiments

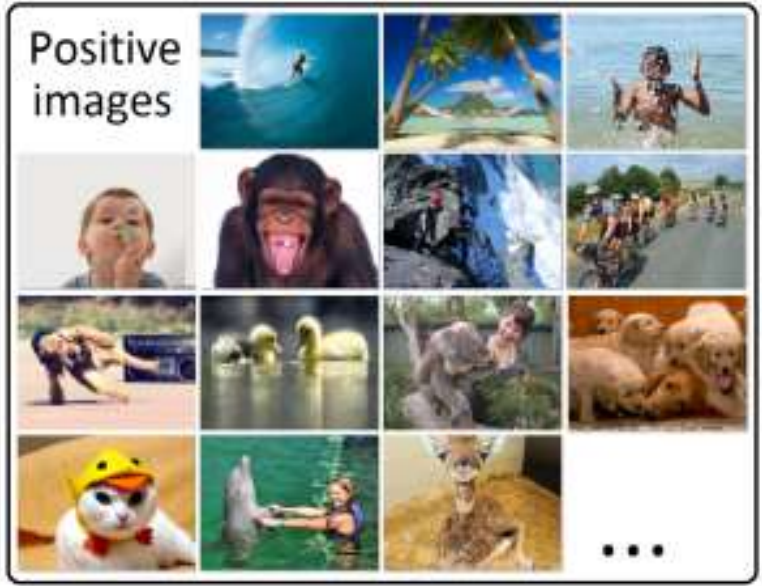

Two wearable sensors were used to measure the skin conductance and heart beat rate of each experimental subject during each session. The skin conductance sensor gave 2 measures per second. The heart beat rate sensor gave 1 measure per second. Researchers took the readings of the wearable sensors during section 2 and section 4 
only. In the duration of each positive/negative image (5 seconds), the sensors provided 10 skin conductance measures and 5 heart beat rate measures, and we only considered the average values of the 5 seconds' measures for higher precision.

A photo taken during one experiment is given in Fig. 8, and the wearable sensors used for the experiments are presented in Fig. 9. A video taken during one experiment session is downloadable from (https://www.dropbox.com/s/dvrazuu0rwkcrfu/videoExperiment_v3_21092015.avi?dl=0). By filtering out the obviously abnormal data points (i.e. no or very low $(<40 \mathrm{bpm})$ heart beat rate, no change in heart beat rate and skin conductance during the whole experiment) due to the failure of wearable sensors, we obtained the data that is downloadable from (https://www.dropbox.com/s/xepili7nwqnemxx/CYBERMIND_Data.rar?dl=0). The details of the data are given in Table 1.

Based on the five days' data, we can partition the experimental subjects' wearable sensor data into data clouds autonomously and objectively [25] to gain a better understanding on how they react to two different sensory input (images and music). Figs. 11 and 12 illustrate the data clouds and the continuous global typicality based on the third and fourth subjects for all the five days during which the experiment took place. Due to the individual differences, the values of PVs measured were centred with the subtracted mean in advance.

One can see that there are differences between the two individuals. On the other hand, the variability between the five days for these individuals is not as much as for the other eight volunteers, Fig. 13 as an example, in which this subject 5 and 6's reactions (emotional states) towards the positive and negative images and music during the

Table 1. Amounts of valid PVs observed per person per day and overall

\begin{tabular}{ccccccc}
\hline \hline & \multicolumn{5}{c}{ Number of valid data sample } \\
Individual \# & Day 1 & Day 2 & Day 3 & Day 4 & Day 5 & Overall \\
\hline 1 & $40 / 40^{\text {a }}$ & $38 / 40$ & $40 / 37$ & $40 / 40$ & $40 / 40$ & $198 / 197$ \\
2 & $40 / 40$ & $40 / 40$ & $40 / 40$ & $38 / 31$ & $40 / 40$ & $198 / 191$ \\
3 & $40 / 40$ & $40 / 40$ & $38 / 39$ & $40 / 39$ & $39 / 40$ & $197 / 200$ \\
4 & $39 / 40$ & $36 / 40$ & $38 / 40$ & $39 / 36$ & $37 / 40$ & $191 / 196$ \\
5 & $0 / 0$ & $40 / 40$ & $38 / 33$ & $39 / 39$ & $40 / 40$ & $157 / 153$ \\
6 & $40 / 40$ & $0 / 0$ & $32 / 40$ & $40 / 40$ & $40 / 40$ & $152 / 160$ \\
7 & $40 / 40$ & $40 / 39$ & $39 / 40$ & $40 / 40$ & $40 / 40$ & $199 / 199$ \\
8 & $40 / 37$ & $39 / 40$ & $39 / 40$ & $40 / 40$ & $40 / 40$ & $198 / 197$ \\
9 & $40 / 40$ & $40 / 40$ & $40 / 40$ & $40 / 40$ & $40 / 40$ & $200 / 200$ \\
10 & $40 / 27$ & $39 / 40$ & $39 / 40$ & $40 / 40$ & $40 / 38$ & $199 / 175$ \\
\hline \hline
\end{tabular}

two days are inverse, and the value ranges are also different. 
Due to the complex influences of living environments, fatigue as well as other factors on human body, there are additional uncertainties. Nonetheless, we found out that, although each person reacted differently and even at each session the same person reacted differently, the data clouds being formed were quite compact and distinct between each other even if moved around or even swapped at some point for some rare cases (Figs. 7 and 13). Therefore, one can conclude that during a particular session of the experiment, a particular volunteer's reactions towards the "positive" and "negative" images and music are highly separable. Moreover, there was no need to label the two emotional states. By forming data clouds (data partitioning) autonomously the classification was made by association with the previous observable PVs data. In this way, the classifier is really autonomous and reflects the sub-conscious nature of the learning taking place in humans.

We conducted additional experiments to test and analyse the results in terms of "Did we achieved what we hoped to achieve - can we cluster the observable PVs into emotions automatically; can we predict what emotions new, unseen images are more likely to invoke". As we stressed in the previous paragraph, by "positive" and "negative", we can consider the two PV pairs that are clustered into different data cloud/cluster. For this purpose, we built a fully autonomous AnYa type self-learning fuzzy rule-based zero order classifier [26] per person per day, and learn to classify the individual's perceptions. The actual human-intelligible labels ("positive" and "negative") require cognitive feedback (see, Fig.1) and are part of the second stage of the 2 hierarchical structure/ architecture (see Fig. 2).

We started by considering the first pair of observable PV data point that corresponds to an image and a piece of music from both categories ("positive" and "negative") to be the sole training data samples. In this way, we consider per person per day a scenario whereby every data sample is given automatically by the classifier a label that is "1" or "2" and it corresponds to the two images and music pieces for which the emotional state (and respectively, the class label) is different, respectively "positive" and" negative". Indeed, the labels are related to what we think about these groups, while grouping similar emotional reactions into clusters/groups and later stating "the current emotional reaction is similar to A or B" where A and B are concepts/data clouds/clusters-like groupings can be done subconsciously and, thus, autonomous. Thus, the classifier predicts the class (or otherwise, the person's reaction) to any future image into one of the two types that were at the very beginning fully autonomously The focal points of the data clouds are formed by self-learning as follows. Initially, the first pair/2D point of PVs data for each data cloud are used and then these are being updated automatically. The focal points are critically important and 
sufficient to form data clouds because they are formed like Voronoi tessellation by assigning each new coming data point to the nearest focal point [24], which means that the system is self-learning to reflect the sub-conscious nature of learning that takes place in humans. Notice, that we do not need to know exactly the label ("positive" or "negative", "good" or "bad", etc.). We simply need to know that they are different, not the same. So, the scenario is fully autonomous. It is per person and per day; that means, not universal "one size fit all", but that is logical humans react differently and also the same human comes next day to the experiment with a psychological baggage.

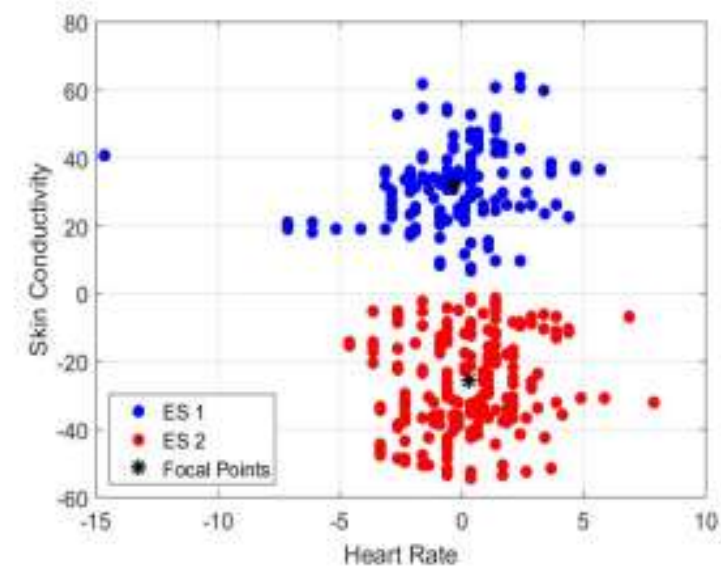

a) Data clouds

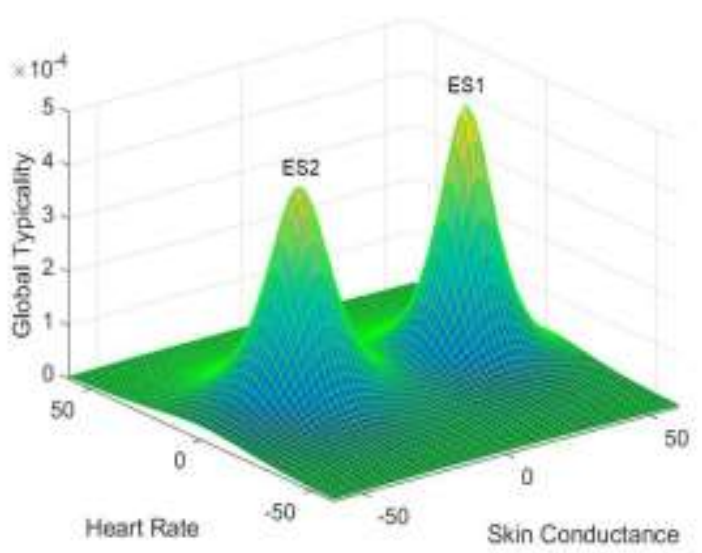

b) Global typicality

Fig. 11. Experimental results for the third individual

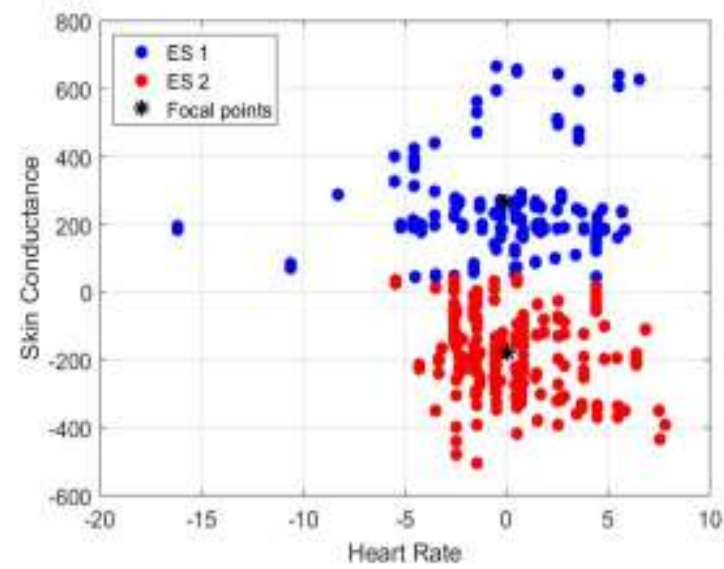

a) Data clouds

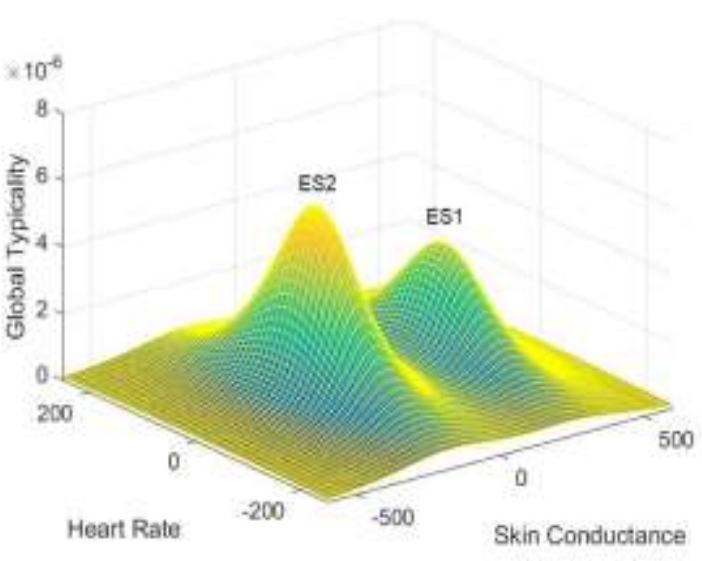

b) Global typicality

Fig. 12. Experimental results for the fourth individual

The results (in the form of confusion matrix) of applying the autonomous data cloud formation method [1] and the AnYa type self-learning fuzzy rule-based zero order classifier are tabulated in Table 2. The corresponding classification accuracies are tabulated in Table 3. The evolution of the fuzzy rules based on the data from individuals 7, 8, 9 and 10 measured on the last day are presented in Table 4, in which we can see that, the final fuzzy 
rules of the $7^{\text {th }}$ and $10^{\text {th }}$ experimental subjects changed in comparison to their initial values based on the first and the only training data pair per class.
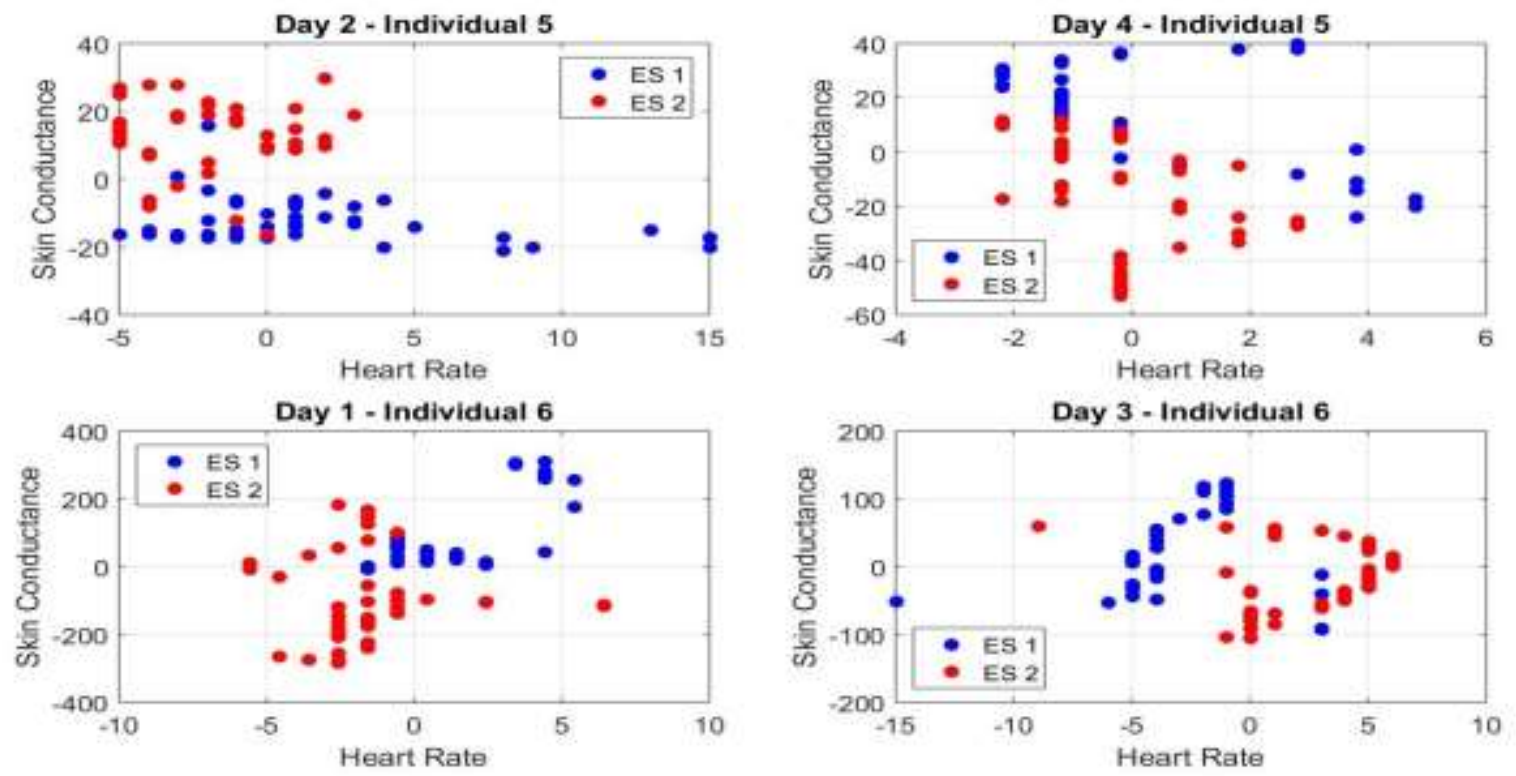

Fig. 13. Differences in reactions of the fifth and sixth individuals during different sessions (days)

Table 2. Confusion matrix of prediction results (per person per day)

\begin{tabular}{|c|c|c|c|c|c|c|c|c|c|c|c|}
\hline \multirow{2}{*}{ 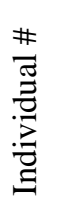 } & & \multicolumn{2}{|c|}{ Day 1} & \multicolumn{2}{|c|}{ Day 2} & \multicolumn{2}{|c|}{ Day 3} & \multicolumn{2}{|c|}{ Day 4} & \multicolumn{2}{|c|}{ Day 5} \\
\hline & Actual & $\bar{\Sigma}$ & $\underset{\widetilde{N}}{\tilde{y}}$ & $\overline{\widetilde{I}}$ & $\underset{N}{\tilde{I}}$ & $\overline{\breve{I}}$ & $\underset{\tilde{c}}{\tilde{I}}$ & $\overline{\tilde{y}}$ & 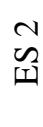 & $\overline{\tilde{y}}$ & N \\
\hline \multirow{2}{*}{1} & Positive & 34 & 5 & 22 & 15 & 3 & 36 & 39 & 0 & 36 & 3 \\
\hline & Negative & 27 & 12 & 2 & 37 & 32 & 4 & 0 & 39 & 9 & 30 \\
\hline \multirow{2}{*}{2} & Positive & 39 & 0 & 39 & 0 & 39 & 0 & 16 & 21 & 39 & 0 \\
\hline & Negative & 0 & 39 & 0 & 39 & 0 & 39 & 29 & 1 & 0 & 39 \\
\hline \multirow{2}{*}{3} & Positive & 39 & 0 & 39 & 0 & 19 & 18 & 39 & 0 & 38 & 0 \\
\hline & Negative & 0 & 39 & 0 & 39 & 19 & 19 & 0 & 38 & 1 & 38 \\
\hline \multirow{2}{*}{4} & Positive & 33 & 5 & 35 & 0 & 24 & 13 & 38 & 0 & 36 & 0 \\
\hline & Negative & 6 & 33 & 4 & 35 & 15 & 24 & 0 & 35 & 3 & 36 \\
\hline \multirow{2}{*}{5} & Positive & & & 38 & 0 & 21 & 16 & 13 & 25 & 39 & 0 \\
\hline & Negative & & & 0 & 35 & 0 & 32 & 0 & 38 & 0 & 39 \\
\hline \multirow{2}{*}{6} & Positive & 33 & 6 & & & 6 & 25 & 39 & 0 & 39 & 0 \\
\hline & Negative & 6 & 33 & 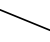 & 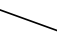 & 8 & 31 & 0 & 39 & 1 & 38 \\
\hline \multirow{2}{*}{7} & Positive & 38 & 1 & 39 & 0 & 25 & 13 & 39 & 0 & 32 & 7 \\
\hline & Negative & 33 & 6 & 0 & 38 & 22 & 17 & 0 & 39 & 29 & 10 \\
\hline \multirow{2}{*}{8} & Positive & 27 & 12 & 7 & 31 & 32 & 6 & 27 & 12 & 39 & 0 \\
\hline & Negative & 33 & 3 & 1 & 28 & 20 & 19 & 6 & 33 & 0 & 39 \\
\hline \multirow{2}{*}{9} & Positive & 36 & 3 & 3 & 36 & 3 & 36 & 23 & 16 & 39 & 0 \\
\hline & Negative & 0 & 39 & 36 & 3 & 24 & 15 & 31 & 8 & 0 & 39 \\
\hline \multirow{2}{*}{10} & Positive & 39 & 0 & 25 & 13 & 32 & 6 & 39 & 0 & 34 & 5 \\
\hline & Negative & 0 & 26 & 23 & 16 & 27 & 12 & 1 & 38 & 30 & 7 \\
\hline
\end{tabular}


The overall classification rate we achieved is around $75 \%$ with some people (individuals $2,3,4,5,7$ ) being "predictable", while some others (individuals $1,8,9$ ) being more complex to predict, especially on some days.

Table 3. Prediction Accuracy (per person per day and overall)

\begin{tabular}{ccccccc}
\hline \hline \multirow{2}{*}{ Individual \# } & Day 1 & Day 2 & Day 3 & Day 4 & Day 5 & Overall \\
\hline 1 & 0.5897 & 0.7564 & 0.0933 & 1.0000 & 0.8462 & 0.6481 \\
2 & 1.0000 & 1.0000 & 1.0000 & 0.2537 & 1.0000 & 0.8458 \\
3 & 1.0000 & 1.0000 & 0.4935 & 1.0000 & 0.9870 & 0.8741 \\
4 & 0.8571 & 0.9459 & 0.6316 & 1.0000 & 0.9600 & 0.8501 \\
5 & & 1.0000 & 0.7681 & 0.6711 & 1.0000 & 0.8226 \\
6 & 0.8462 & & 0.5286 & 1.0000 & 0.9870 & 0.8269 \\
7 & 0.5641 & 1.0000 & 0.5455 & 1.0000 & 0.5385 & 0.7111 \\
8 & 0.4000 & 0.5224 & 0.6623 & 0.7692 & 1.0000 & 0.6430 \\
9 & 0.9615 & 0.0769 & 0.2308 & 0.3924 & 1.0000 & 0.5200 \\
10 & 1.0000 & 0.5325 & 0.5714 & 0.9872 & 0.5395 & 0.7166 \\
\hline \hline
\end{tabular}

Table 4. Evolution of the AnYa type fuzzy rules

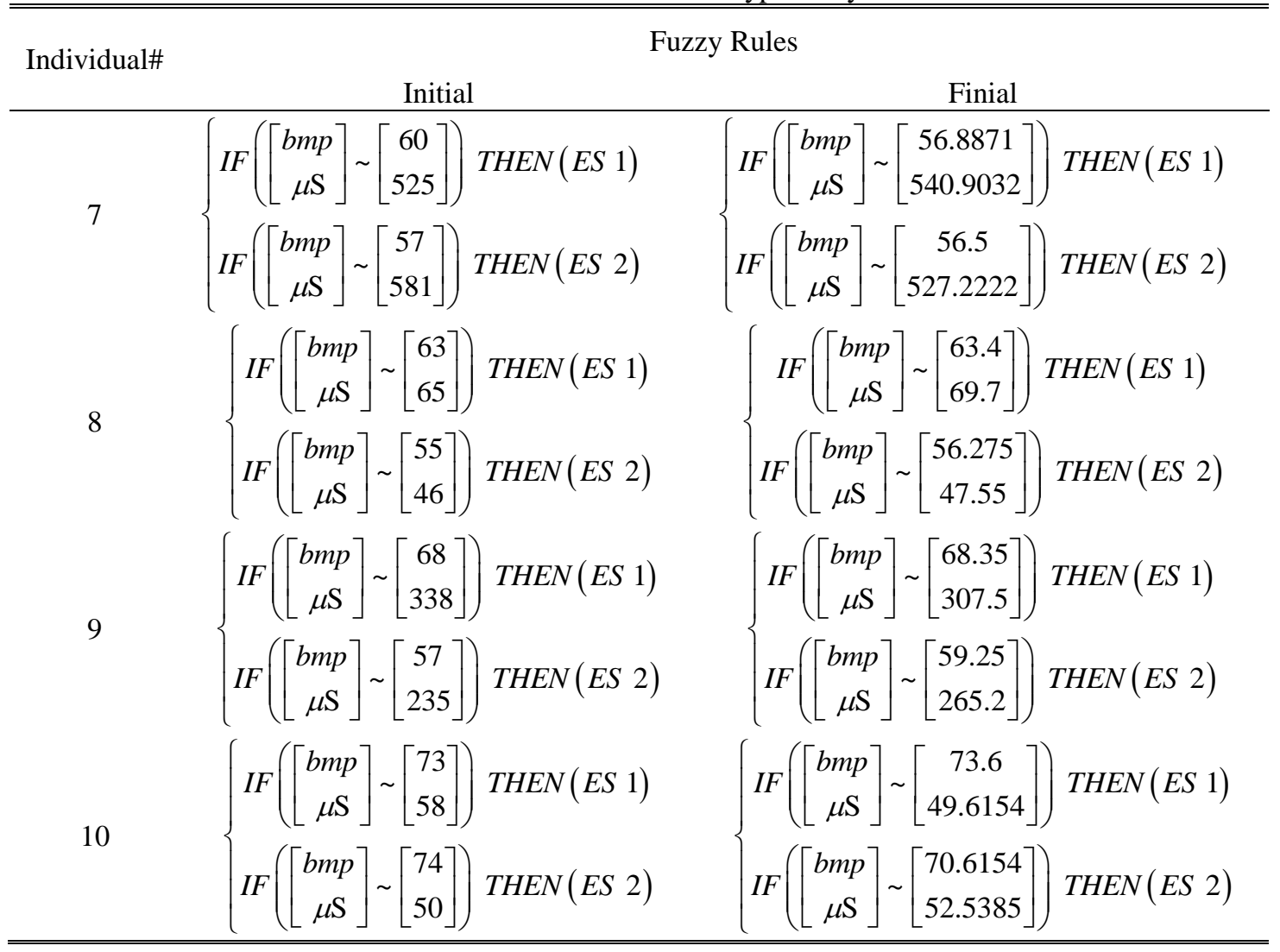

Firstly, all the predictions were conducted based on only two features (heart beat rate and skin conductance), but human body is very complex, two features are far from enough to properly describe the human reactions. As a result, we need to further study the human bodies and collect more related data. 
Secondly, in our experiments, the environments and other factors that may influence the results were neglected in order to simplify the modelling process. However, those factors are very important, for example, if someone washed his hands before experiments, his skin resistance could be largely changed.

Moreover, in many cases, the wearable sensors as we used in the experiments failed to give very reliable readings. This problem can be solved by using more precise sensory devices.

\section{Conclusion}

In this paper we study and experiment in laboratory environment with real individuals about the learning of individual's perceptions to visual and auditory (and we extrapolate to the other three types of sensory) inputs. We argue that the perceptions and reactions are individual as well as context and time-dependent, however, are predictable and stable during a given session. Moreover, we propose a systematic mathematical and computational cybernetic model as well as a fully autonomous methodology to cluster and classify the emotional expression of the individual's perceptions by using the recently introduced empirical data analytics computational framework. We simplify the real experiments focusing on two observable/measurable physical variables (PVs): heart beat rate measured using a wearable low cost sensor and skin conductance (also measured by a wearable low cost sensor), and thus, we consider the similarity between PVs and, respectively, emotions. As a result, we form fully automatically, online and in real-time data clouds (cluster-like sets of data) that corresponds to the two emotional states (positive and negative feelings). Furthermore, without explicitly using any label, a set of IF ...THEN ... type self-evolving fuzzy rule-based classifiers of zero order is proposed and developed which is able to classify the emotional state for a given measurement of PVs (these correspond to images and audio signal/music). Due to the fact that, despite that each person reacted differently and even at each session differently, the data clouds formed empirically from the observed PVs were quite obviously compact and yet distinct between each other even if moved around or even swapped at some point for some rare cases, we were able to achieve very encouraging results: for this extremely complicated problem, around $75 \%$ correct classification results is obtained without any pre-training. Indeed, the proposed classifier is autonomously self-learning to reflect the sub-conscious nature of learning that takes place in humans. For each measurement pair of the PVs the most likely class is assigned based on similarity and proximity in the data space while the labels are not important (e.g. emotional reaction type "1" and "2" or same reaction as the first measurement or a different one and so on. Indeed, human-intelligible labels "positive" or 
"negative", "good" or "bad" assume conscious involvement of a decision, while it is well-known that humans can associate images, sounds, etc., to previously seen ones even if the labelling may be difficult, questionable or impossible. Such an autonomous classifier as the proposed one can also be used as a kind of a lie detector or identifier of emotional reactions even if the individual is not actively taking part by labelling them - the emotional reaction will actually speak for itself and provide the ground truth. The proposed classifier automatically groups similar emotional reactions into data clouds and associates the current emotional reaction with the concept identified previously by autonomous data partitioning (clustering-like procedure). The proposed methodology can also be used to build and evaluate quantitatively emotional and psychological profiles of people and professionals working in stressful or responsible situations, for medical and advertising purposes. Of course, this can only end in grouping the same types of reaction and will not ultimately place a human intelligible label. However, the result can be interpreted as "IF (the values of the observable PVs are similar) THEN (the images, audio/music invoke in this particular individual during this session the same type of reaction)". The labels that remain to be placed are the same across all individuals and all days of experimental sessions. In this way, the only input if necessary is to actually place the labels of "positive" and "negative" once. This type of a fully autonomous classifier is quite new (in fact, we recently published a method for fully autonomous FDI (fault detection and identification) which is based on similar principles) and we argue is quite suitable to represent the way people actually learn. That is, we argue that unsupervised learning is quite important form of mapping the world we live in into human perceptions, emotions and reactions (the sub-conscious aspect).

\section{References}

[1] M. Meredith and B. Stein, "Interactions among converging sensory inputs in the superior colliculus," Science, vol. 221, no. 4608, pp. 1981-1983, 1983.

[2] B. Stein and T. Stanford, et al., "The neural basis of multisensory integration in the midbrain: Its organization and maturation," Hear. Res., vol. 258, no. 1, pp. 4-15, 2009.

[3] T. Koelewijn and A. Bronkhorst, et al., "Attention and the multiple stages of multisensory integration: A review of audiovisual studies," Acta Psychol. (Amst)., vol. 134, no. 3, pp. 372-384, 2010.

[4] C. Cappe and E. Rouiller, et al., "Multisensory anatomical pathways," Hear. Res., vol. 258, no. 1, pp. 28-36, 2009.

[5] L. Zadeh, "Fuzzy sets," Inf. Control, vol. 8, no. 3, pp. 338-353, 1965. 
[6] L. Itti and C. Koch, "Computational modelling of visual attention.," Nat. Rev. Neurosci., vol. 2, no. 3, pp. 194-203, 2001.

[7] M. Poel and R. Akker, et al., "Learning Emotions in Virtual Environments," Proc. Sixt. Eur. Meet. Cybern. Syst. Res., pp. 751-756, 2002.

[8] F. Celli and E. Bruni, et al., "Automatic Personality and Interaction Style Recognition from Facebook Profile Pictures," Proc. ACM Int. Conf. Multimed. - MM '14, pp. 1101-1104, 2014.

[9] E. Tighe and J. Ureta, et al., "Personality Trait Classification of Essays with the Application of Feature Reduction.," in 25th International Joint Conference on Artificial Intelligence IJCAI, 2016, pp. 1-5.

[10] J. Principe and R. Chalasani, "Cognitive architectures for sensory processing," Proc. IEEE, vol. 102, no. 4, pp. 514-525, 2014.

[11] T. Huang and S. Grossberg, "Cortical dynamics of contextually cued attentive visual learning and search: spatial and object evidence accumulation.," Psychol. Rev., vol. 117, no. 4, pp. 1080-1112, 2010.

[12] P. Angelov and R. Yager, "A new type of simplified fuzzy rule-based system," Int. J. Gen. Syst., vol. 41, no. 2, pp. 163-185, 2011.

[13] T. Hastie and R. Tibshirani, et al., The Elements of Statistical Learning: Data Mining, Inference, and Prediction, Second Edition. 2009.

[14] P. Angelov, Autonomous Learning Systems: From Data Streams to Knowledge in Real Time. John Willey, 2012.

[15] J. Andreu and R. Baruah, et al., "Automatic scene recognition for low-resource devices using evolving classifiers," IEEE Int. Conf. Fuzzy Syst., pp. 2779-2785, 2011.

[16] X. Gu and P. Angelov, et al., "Forming data clouds: free-shape data partitioning through empirical data analysis," Submitt. to IEEE Trans. Neural Networks Learn. Syst.

[17] P. Angelov, "Anomaly detection based on eccentricity analysis," in 2014 IEEE Symposium Series in Computational Intelligence, IEEE Symposium on Evolving and Autonomous Learning Systems, EALS, SSCI 2014, pp. 1-8.

[18] A. Coenen and W. Drinkenburg, "Animal models for information processing during sleep," Int. J. Psychophysiol., vol. 46, no. 3, pp. 163-175, 2002.

[19] M. Schredl and D. Atanasova, et al., "Information processing during sleep: The effect of olfactory stimuli on 
dream content and dream emotions," J. Sleep Res., vol. 18, no. 3, pp. 285-290, 2009.

[20] R. Picard and E. Vyzas, et al., "Toward machine emotional intelligence: analysis of affectivelnphysiological state," IEEE Trans. Pattern Anal. Mach. Intell., vol. 23, no. 10, pp. 1175-1191, 2001.

[21] P. Angelov, "Outside the box: an alternative data analytics framework," J. Autom. Mob. Robot. Intell. Syst., vol. 8, no. 2, pp. 53-59, 2014.

[22] P. Angelov, "Typicality distribution function - a new density- based data analytics tool," in IEEE International Joint Conference on Neural Networks (IJCNN), 2015, pp. 1-8.

[23] P. Angelov and X. Gu, et al., "Empirical data analysis - a new tool for data analytics," in IEEE International Conference on Systems, Man, and Cybernetics, 2016, pp. 53-59.

[24] P. Angelov and X. Gu, et al., "A generalized methodology for data analysis,” IEEE Trans. Cybern., 2017, to appear.

[25] P. Angelov and X. Gu, "Local Modes-based Free-Shape Data Partitioning," in IEEE Symposium Series on Computational Intelligence, IEEE Symposium on Evolving and Autonomous Learning Systems, 2016, pp. 18.

[26] P. Angelov and X. Gu, et al., "Autonomous Learning Multi-model Systems from Data Streams," IEEE Trans. Fuzzy Syst., 2017, to appear. 